\title{
MODELAGEM DO PERFIL TRANSVERSAL DE RESISTÊNCIA À FLEXÃO EM MÁQUINA DE PAPEL COM REDES NEURAIS
}

\author{
CROSS DIRECTION BENDING STIFFNESS PROFILE \\ MODELLING IN PAPER MACHINE WITH NEURAL \\ NETWORKS
}

\author{
Luiz Renato Pesch* \\ Ivo Neitzel ${ }^{* *}$
}

\begin{abstract}
RESUMO
A resistência à flexão (rigidez) é uma característica importante de um papel cartão, pois define a capacidade de utilização do papel cartão em embalagens dos mais diversos produtos. Ela não é constante ao longo do perfil transversal à máquina de papel pelo fato de que é medida após a folha estar pronta. $\mathrm{O}$ objetivo do trabalho foi a obtenção de um modelo a partir de redes neurais e utilizando variáveis de máquina que permita predizer o valor da resistência à flexão. As variáveis adotadas foram a gramatura, espessura e umidade divididas nos setores onde havia medição da resistência à flexão pelo laboratório. Existem onze setores, o que levou a construção de onze redes neurais com duas saídas cada uma - uma para a resistência à flexão no sentido longitudinal da folha e a outra para o sentido transversal. Não foi adotado um papel cartão específico para a modelagem, utilizaram-se todos os produzidos. Os modelos obtidos com as redes apresentaram bons resultados, baixos erros relativos, que indicam uma grande capacidade de generalização, mesmo adotando-se uma gama grande de produtos. O que mostra que é possível a construção de um sensor virtual utilizando redes neurais que disponibilizam ao operador da máquina de papel o perfil transversal de resistência à flexão.
\end{abstract}

Palavras-chave: Máquina de papel. Resistência à flexão. Perceptron multicamadas. Redes neurais artificiais.

\begin{abstract}
Bending stiffness is an important characteristic of paperboard as it defines the capacity to use paperboard for packaging various products. Bending stiffness is not constant over the cross section of the paper machine because it is measured after the sheet is finished. The aim of this study was to obtain a model using neural networks and machine variables able to predict bending stiffness. The variables
\end{abstract}

\footnotetext{
*Mestrado em Engenharia Química pela Universidade Estadual de Maringá. Professor da Faculdade de Telemaco Borba.

${ }^{* *}$ Doutorado em Engenharia Química pela Universidade Federal do Rio de Janeiro. Professor Pesquisador da Faculdade de Telêmaco Borba.
} 
adopted were the basis weight, thickness and moisture divided in sectors where there were measurements of bending stiffness. There are eleven sectors, which led to the construction of eleven neural networks with two outputs each - one for the bending stiffness in the machine direction of the sheet and another for the cross direction. All types of paperboard were used for modelling. The models obtained with the networks showed good results which indicate a great generalization capacity, even adopting a wide range of products, showing that it is possible to build a virtual sensor using neural networks.

Keywords: Paper Machine. Bending stiffness. Perceptron multilayer. Artificial neural networks.

\section{Introdução}

A resistência à flexão é uma variável de grande importância em papel cartão, pois ele é utilizado em embalagens, onde resistência é fundamental. Normalmente a medição é efetuada em laboratório, seu valor é obtido após o cartão estar produzido.

O cartão não apresenta uma estrutura homogênea, o que implica que no perfil transversal à máquina encontraremos diferentes valores. Normalmente ela é maior na região central da folha. Nesta condição, os ajustes que se fizerem necessários para se obter os valores objetivos só aparecerão no cartão que irá ser produzido, o que poderá representar uma perda do que já está pronto.

A finalidade do presente trabalho foi investigar a possibilidade da criação de um modelo de predição da resistência à flexão que possa fornecer o perfil transversal à máquina. Com um modelo de predição pode-se criar um sensor virtual que disponibilizaria os valores de rigidez durante a produção do papel cartão.

Para a modelagem, foram utilizadas redes neurais e como variáveis de entrada a gramatura, espessura e umidade medidas pelos sensores de perfil transversal.

\section{Fundamentação teórica}

\subsection{Papel cartão}

Segundo a Associação Brasileira de Celulose e Papel (BRACELPA):

"papel cartão é um produto resultante da união de várias camadas de papel e sobrepostas, iguais ou distintas, que se aderem por compressão. Nessas camadas podem ser utilizada celulose virgem (recur- sos renováveis) e materiais celulósicos recicláveis. Independente do tipo, o papel cartão é fabricado na faixa de gramatura de 200 a $500 \mathrm{~g} / \mathrm{m}^{2}$, com ou sem revestimento superficial."

KIVIRANTA (2000) considera que um papel é um papel cartão quando a gramatura é superior a $150 \mathrm{~g} / \mathrm{m}^{2}$, aceitando exceções com gramaturas abaixo de $100 \mathrm{~g} / \mathrm{m}^{2}$.

Papel é uma rede estocástica de fibras. Desde que as fibras são mais longas que a espessura da folha, a rede é considerada planar e quase bidimensional. A estrutura bidimensional governa muitas propriedades do papel, mas a estrutura porosa tridimensional também é importante. $\mathrm{O}$ caminho produzido pelos poros determina como fluidos são transportados através da folha (NISKANEN, PAKARINEN, 2000). As características físicas do papel são avaliadas em duas direções relativas à máquina de papel. A direção longitudinal é a direção que a folha percorre na máquina e a direção transversal é a direção perpendicular à longitudinal. A direção afeta algumas características físicas do papel.

A BRACELPA faz a classificação dos cartões em função de sua forma construtiva, sendo os mais comuns:

DUPLEX: possui a superfície branca e apresenta miolo e verso escuros, utilizado geralmente em embalagens de sabão em pó medicamentos, cereais, gelatinas, mistura para bolos, caldos, biscoitos e brinquedos.

TRIPLEX: a superfície e verso são brancos e miolo escuro, normalmente utilizado em embalagens de chocolates, cosméticos, medicamentos, fast food, caixas de bombons e bebidas.

SÓLIDO: apresenta todas as suas camadas 
brancas. Usado em embalagens de cigarros, cosméticos, medicamentos, higiene pessoal, fast food, capas de livros e cartões postais.

\subsection{Requisitos mecânicos}

O papel cartão é utilizado como embalagem dos produtos normalmente na forma de caixas dobradas, o que o leva a ter como requisito básico certo nível de resistência mecânica e rigidez. As caixas podem ser empilhadas, e por isto é necessária uma suficiente resistência à compressão (KAJANTO, 2000).

A resistência à flexão, e especialmente a resistência à flexão transversal (direção transversal à máquina de papel), é crítica. A resistência à flexão, rigidez é afetada principalmente pela espessura do papel cartão e o módulo de elasticidade é dado pela matéria-prima contida nele. A estrutura ótima é aquela que possui pelo menos três camadas, sendo a camada do meio volumosa e as camadas de cobertura e base com um grande módulo de elasticidade. Deve-se buscar a menor gramatura possível para uma dada rigidez, conseguindo-se assim maior área produzida pela mesma massa de fibras (KAJANTO, 2000).

Mesmo se a estrutura do cartão for assimétrica, como em revestimento de um lado, a rigidez é quase sempre simétrica. O mesmo momento é necessário na flexão em direção à base como em direção à cobertura, desde que os planos de carga em toda a folha se mantenham na região elástica linear. A resistência à flexão é simétrica porque o módulo de elasticidade é o mesmo para a tração e compressão (KAJANTO, 2000).

Algumas vezes, a rigidez parece ser assimétrica por causa do encanoamento da amostra e é maior quando a flexão ocorre ao longo do eixo de encanoamento na direção do lado convexo da folha. $\mathrm{O}$ encanoamento pode ser tão fraco que o próprio peso da amostra a mantém plana, e mesmo quando não visto, pode ainda afetar a resistência à flexão.

Em grandes flexões, os esforços locais dentro da folha podem crescer até a região não linear da curva de carga, então a direção da flexão torna-se relevante. $\mathrm{O}$ lado côncavo comprimido é o primeiro a ir para a região não linear, por isso uma folha assi- métrica apresenta uma baixa rigidez quando fletida excessivamente em direção ao seu lado mais fraco. A não linearidade na compressão pode ser reversível, e a amostra pode não ter uma mudança permanente na curvatura mesmo quando entrar na região não linear (KAJANTO, 2000).

\subsection{Problemas práticos relacionados à rigidez}

Quando a rigidez causa problemas, usualmente, o motivo é a rigidez transversal que é tipicamente duas a quatro vezes menor que a longitudinal. A anisotropia aparece por causa da orientação das fibras e o encolhimento na direção transversal que ocorre na secagem que afetam o módulo de elasticidade. A importância da anisotropia na rigidez depende do uso final do cartão. $\mathrm{Na}$ impressão, normalmente é crítica a rigidez longitudinal, mas problemas de andamento de máquina também aparecem se a rigidez transversal diminuir muito. Na fabricação de caixas, o fator crítico é a baixa rigidez, independendo da direção, favorecendo um baixo grau de anisotropia (KAJANTO, 2000).

Existem três possibilidades para se aumentar a rigidez (KAJANTO, 2000):

Aumentar a espessura do papel: o maior problema é alcançar uma superfície lisa para a impressão

Módulo de elasticidade mais alto: não é possível com ações que reduzam a espessura

Módulo de elasticidade mais alto nas superfícies que no meio da folha: se cada camada superficial for 5\% da espessura do cartão, então dobrando seus módulos de elasticidade aumenta a rigidez em $27 \%$.

A última possibilidade é a utilização de estrutura em camadas, que é o meio mais efetivo para se obter uma alta rigidez com um bom acabamento superficial e uma baixa gramatura. Uma estrutura em camadas pode ser obtida pela colocação de finos (fibras com comprimento pequeno que não são retidas pela tela formadora da folha) na superfície durante a drenagem, com formação estratificada usando somente uma caixa de entrada e colagem superficial quando a cola não penetra a folha. $\mathrm{O}$ revestimento modifica o módulo de elasticidade da superfície da folha, mas dependendo da gramatura do cartão pode ter um efeito muito pequeno (KAJANTO, 2000). 


\subsection{Efeitos da fabricação na rigidez}

Cada tipo de polpa empregado tem seu potencial de rigidez, mas o valor final no cartão é baixo por causa das condições da máquina de papel e os tratamentos de conversão. A propriedade mais importante da polpa é o volume (bulk), sendo menos importante o módulo específico de elasticidade, obtido pela divisão do módulo de elasticidade pelo peso específico do corpo de prova (CASTANHO e OLIVEIRA, 2000). O efeito da polpa pode ser caracterizado da seguinte forma (KAJANTO, 2000):

Fibras flexíveis proporcionam papel denso e baixa rigidez;

Fibras rígidas proporcionam papel volumoso e alta rigidez;

Ligações rígidas fornecem papel com alta resistência à flexão.

Um bom exemplo de efeitos contrários da habilidade de ligação e flexibilidade da fibra é a refinação que aumenta os dois e por isto tem um grande incremento no módulo de elasticidade. Desde que fibras flexíveis também diminuam a espessura do cartão, a rigidez pode não se alterar (KAJANTO, 2000).

A seção de formação da máquina de papel tem grande influência nas variações na direção z da estrutura do cartão - particularmente na distribuição de finos. Estas distribuições, por sua vez, determinam a rigidez. Muda-se a anisotropia da rigidez alterando-se a orientação das fibras, deformações úmidas (prensagem com a folha úmida) e tensões na secagem que em grandes valores aumentam a rigidez longitudinal, mas tem pouco efeito na transversal. Um aumento na anisotropia da orientação de fibras provoca um aumento na rigidez longitudinal, mas diminui a transversal. O aumento do encolhimento da folha durante a secagem diminui a rigidez transversal principalmente nas bordas da folha (KAJANTO, 2000).

Na prensagem a úmido, o grau de ligação do cartão aumenta, mas a densidade diminui e a rigidez diminui. Conforme SZIKLA e PAULAPURO, citados por KAJANTO (2000), algumas vezes a prensagem úmida pode ser ajustada para melhorar a rigidez através da distribuição da densidade do papel na direção $z$.

O principal efeito da calandragem na rigidez provém da diminuição da espessura. Em calandragem pesada, o módulo específico de elasticidade também diminui. OVASKA, citado por KAJANTO (2000), indica que o módulo específico de elasticidade pode também aumentar se a umidade no papel exceder a $10 \%$ na calandragem. Isso atua no sentido contrário do efeito negativo da redução de espessura.

\subsection{Medição da rigidez}

A medição de rigidez é feita seguindo o procedimento da TAPPI T 556 PM-95(1995). Este procedimento é usado para se medir a rigidez do cartão no sentido longitudinal e transversal, pela determinação da resistência a flexão em $\mathrm{mN}$ de uma amostra de $38 \mathrm{~mm}$ de largura verticalmente presa por um dos lados, em uma deflexão padrão de 15 graus.

\section{Metodologia}

\subsection{Caracterização do material}

O presente trabalho utilizou como fonte geradora dos dados cartões, classificados como do tipo DUPLEX com três camadas, com e sem revestimento e com a rigidez média geométrica variando numa faixa de $80 \mathrm{mN} .50 \mathrm{~mm}$ a $800 \mathrm{mN} .50 \mathrm{~mm}$

\subsection{Escolha das variáveis de processo para modelagem}

As variáveis que possuem medição em linha no perfil transversal são a espessura, a gramatura e a umidade. Como a rigidez pode ser expressa pela espessura e pela gramatura, estas variáveis podem representar bem o comportamento do perfil de rigidez. A umidade tem um impacto negativo no módulo de elasticidade, o que pode ajudar a caracterizar o processo.

A maior dificuldade aparece para relacionar os pontos medidos do sensor com os pontos de rigidez medidos pelo laboratório. O sensor divide o perfil em 360 pontos, com cada ponto cobrindo aproximadamente $18 \mathrm{~mm}$, e são mapeados na largura da caixa de entrada da máquina. Como existe o corte das laterais na região de formação e o encolhimento da folha na secagem, a largura da folha no sensor será menor que os 360 pontos que ele pode medir. A largura da folha no sensor estará entre $6100 \mathrm{~mm}$ e $6200 \mathrm{~mm}$. 
A amostragem é feita dividindo-se a folha em 16 partes e tomando-se 11 para a medição de rigidez.

A figura 1 mostra como é feita a retirada das amostras para a análise de rigidez. Cada amostra vai gerar dois corpos de prova, um para a rigidez longitudinal e o outro para a transversal. Como cada corpo de prova tem $38,1 \mathrm{~mm}$ por $80 \mathrm{~mm}$, então para a caracterização da rigidez serão necessários três pontos do sensor para a longitudinal e cinco pontos para a transversal.

A tabela 1 mostra como foram adotados os pontos do sensor para cada setor de medição da rigidez. O início da varredura do sensor é do lado de acionamento da máquina que corresponde ao setor 11 da medição de rigidez. Foi considerado para as folhas mínima e máxima, que cada amostra teria 10 pontos do sensor e iniciando a $20 \mathrm{~mm}$ da borda mais próxima. Os pontos do sensor adotados para cada setor de rigidez deveriam estar dentro do intervalo de início da folha mínima e final da folha máxima e em número de 11. Para efeito da tabela, foi considerado que a origem do sensor está no início da folha. Assim, para cada variável medida pelo sensor, serão utilizados 11 pontos por setor para garantir que as variações na amostragem estão incluídas na base de dados, o que leva a ter 33 entradas para cada saída da rede neural.

\subsection{Base de dados e pré-processamento}

Os dados de processo e laboratório são mantidos num historiador (Plant Information - OSISoft, Inc.) o que facilita muito a construção da base de dados para o treinamento da rede neural. As variáveis utilizadas foram as de rigidez por setor, a espessura, a gramatura, e a umidade. Foram incluídas ainda na base variáveis que auxiliam na verificação da consistência dos dados, como o tipo de produto e o estado da máquina. A base foi construída com 396.000 linhas de dados com intervalo entre as medições de 1 minuto.

A primeira parte da limpeza de dados consistiu da retirada dos valores que existiam quando a máquina de papel estava parada ou com o rompimento da folha de papel. Após, foi verificado o comportamento dos valores fornecidos pelos sensores para ver se eles estavam apresentando condição que indicasse falha ou sensor desativado. Verificou-se o valor atual e comparou-se com o anterior, se a diferença foi menor que $2 \%$, colocou-se o valor atual em condição de erro.

A amostra retirada do rolo para o teste da rigidez é feita no fim do rolo e tem associado o horário da finalização do rolo. Este horário foi utilizado para os valores fornecidos pelos sensores para cada variável, os outros valores fora deste horário foram descartados. A próxima etapa consistiu na verificação da consistência dos dados em relação ao produto sendo produzido. Após estas etapas a base de dados estava pronta para o inicio da modelagem.

\subsection{Modelagem da rigidez}

A modelagem da rigidez foi feita utilizando-se um produto da Pavilion Technologies, PROPERTY PREDICTOR. Com ele, pode-se criar uma rede do tipo MLP retro propagação. Outra vantagem dele é o módulo de pré-processamento que possui muitos recursos facilitando a limpeza da base de dados.

Para a modelagem do perfil, adotaram-se redes do tipo MLP retro propagação com função de ativação sigmoidal e 15 neurônios na camada intermediária. Foram criadas 11 redes, uma para cada valor medido pelo laboratório. Cada rede tendo 33 entradas (11 medidas de espessura, 11 de gramatura de fibras base seca e 11 de umidade) e 2 saídas (rigidez longitudinal e rigidez transversal).

O primeiro treinamento de cada rede foi feito com curta duração com o intuito de verificar se a base de dados realmente estava consistente. Utilizando-se um gráfico de predito x medido, os pontos que caiam fora da faixa de três desvios padrão foram novamente verificados para ver se realmente eram válidos. Após esta validação, o treinamento foi executado com a finalidade de se obter um modelo do comportamento da rigidez em função de três variáveis escolhidas.

Todos os treinamentos foram feitos com restrições nas variáveis. Como se sabe que a rigidez tem relação direta com a gramatura e a espessura só foi aceita esta relação no treinamento. A umidade reduz o módulo de elasticidade, logo, ela tem uma relação inversa com a rigidez. Assim foi adotada esta relação no treinamento. 


\subsection{Análise dos resultados}

A análise dos resultados foi feita utilizando-se as ferramentas disponíveis no PROPERTY PREDICTOR.

A avaliação foi baseada no erro relativo que indica a discrepância entre o valor real da saída na base de dados e o valor predito da saída gerado pelo modelo.

Um erro relativo, se computável, é um número real maior que zero. Um erro relativo de zero indicaria que o modelo pode predizer perfeitamente a saída a partir das entradas. Um erro relativo com valor 1 indicaria que o modelo prediz tão bem quanto predizendo a média dos dados. Geralmente, um modelo com um erro relativo menor que 0,8 pode ser utilizado (PAVILION,1999).

O erro relativo é computado em três níveis:

- Para variáveis de saída como uma composição para cada padrão: rel_err

- Para os padrões como uma composição de toda base de dados: rel_err ${ }_{\text {tot }}$

- Para se calcular os erros relativos utiliza-se o quadrado dos erros. A equação (1) é usada para se computar o quadrado do erro para uma variável de saída individual

$$
s q_{-} e r r_{\text {out }}=\left(y_{\text {out }}-\hat{y}\right)^{2}
$$

onde

$\mathrm{y}_{\text {out }}$ é o valor real para a variável de saída no padrão e $\hat{y}$ é o valor de saída pelo modelo.

A equação (2) calcula o quadrado do erro para um padrão, que é a soma dos quadrados dos erros para cada saída no padrão

$$
s q_{-} e r r_{\text {pat }}=\sum_{\text {out }=1}^{N_{\text {outs }}} s q_{-} e r r_{\text {out }}
$$

onde

out é o índice de saída e $\mathrm{N}_{\text {outs }}$ é o total de saídas no padrão.

O quadrado dos erros para a base de dados, equação (3), é a soma do quadrado dos erros para cada padrão na base de dados.

$$
s q_{-} e r r_{t o t}=\sum_{p a t=1}^{N_{p a t s}} s q_{-} e r r_{p a t}
$$

onde

pat é o índice do padrão e $\mathrm{N}_{\text {pats }}$ é o número total de padrões na base de dados.

O erro relativo para uma variável de saída individual, rel_err ${ }_{\text {out, }}$ é calculado pela equação (4).

$$
r e l_{-} e r r_{\text {out }}=\sqrt{\frac{s q_{-} e r r_{\text {out }}}{\sigma_{\text {out }}^{2}}}
$$

onde

$\sigma_{\text {out }}^{2}$ é a variância dos valores reais da saída. O desvio padrão para uma saída é computado de todos os valores para uma variável de saída na base de dados durante o treinamento.

A equação (5) fornece o erro relativo de um padrão $\left(\right.$ rel_err $\left.{ }_{\text {pat }}\right)$.

$$
r e l_{-} e r r_{\text {pat }}=\sqrt{\frac{s q_{-} e r r_{\text {pat }}}{N_{\text {outs }} \times \sigma_{\text {all_outs }}^{2}}}
$$

onde

$$
\sigma_{\text {all_outs }}^{2}=\frac{1}{N_{\text {outs }}} \sum_{\text {out } 1}^{N_{\text {outs }}} \sigma_{\text {out }}^{2}
$$

O erro relativo para uma base de dados inteira (rel_err ${ }_{\text {tot }}$ ) é dado pela equação (7). 


$$
r e l \_e r r_{\text {tot }}=\sqrt{\frac{s q_{-} e r r_{\text {tot }}}{N_{\text {pats }} \times N_{\text {outs }} \times \sigma_{\text {all_outs }}^{2}}}
$$

$\mathrm{O}$ erro relativo não tem o mesmo significado que a medida estatística comumente usada, $\mathrm{R}^{2}$. Se o erro relativo for menor que um, as duas medidas podem ser relacionadas da seguinte forma:

$$
R^{2}=1-r e l \_e r r^{2}
$$

Outra forma de avaliação do desempenho das redes neurais é a verificação da dispersão dos dados em um gráfico que possui no eixo das ordenadas os valores das medições e no eixo das abscissas os valores preditos pelas redes neurais. Quanto maior for a dispersão menor a capacidade da rede de predizer com precisão.

\section{Resultados e discussões}

Nesta seção, serão apresentados os resultados obtidos nos treinamentos das redes. A gramatura usada contempla somente a gramatura de fibras, descontada a umidade e revestimento.

Foram utilizados em média 4200 padrões para o treinamento, sendo divididos da seguinte forma: 3300 para o treinamento propriamente dito, 255 para validação e o restante para o teste do treinamento. A fase de teste do treinamento serve para balizar o desempenho do treinamento. A etapa de validação é feita com dados não utilizados pela rede e serve para se comprovar o desempenho dela.

A tabela 2 apresenta os resultados dos treinamentos das 11 redes. Na coluna "Todos", são mostrados os valores dos erros relativos, de $\mathrm{R}^{2}$, e do desvio padrão feitos com todos os padrões disponíveis, inclui os padrões de validação e teste. Na coluna "validação", o erro mostrado é só o dos padrões de validação.

Avaliando-se $\mathrm{R}^{2}$ de todas as redes pode-se dizer que todas elas possuem um alto grau de correlação com a rigidez. O erro relativo apresenta valores baixos o que comprova o bom desempenho das redes. E, de forma geral, a rigidez longitudinal apresenta uma correlação levemente melhor que a rigidez transversal.
Avaliando-se os erros relativos e $\mathrm{R}^{2}$ da validação, pode-se dizer que as redes apresentaram um bom desempenho para padrões desconhecidos. De forma geral para estes padrões houve uma melhora para a rigidez longitudinal e uma piora para a transversal, mas ainda apresenta uma boa correlação.

Os gráficos 1 a 6 mostram a dispersão dos dados obtidos na medição (ordenadas) em relação aos valores preditos (abscissas) para as redes laterais e a central.

Avaliando-se os gráficos, existe uma região em todos eles que esta fora de três desvios padrão (a região de alta rigidez), isto indica que nesta região a rede não consegue descrever bem o processo, provavelmente porque existe uma menor quantidade de padrões nesta região para o treinamento.

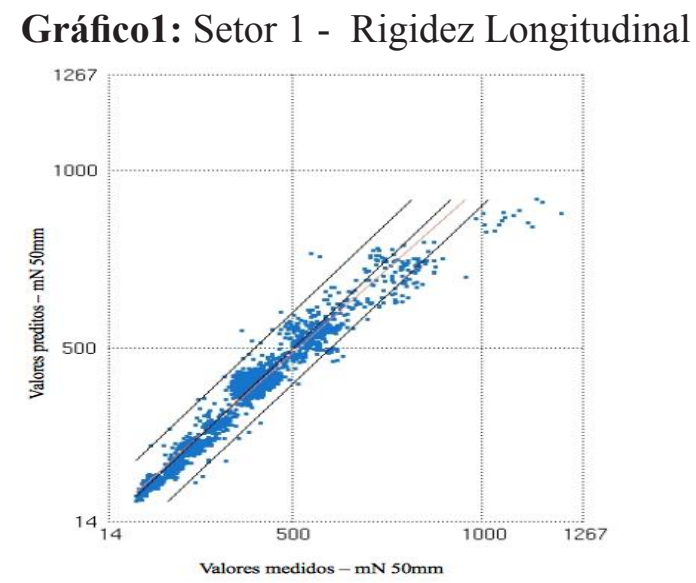

Gráfico 2: Setor 1- Rigidez Transversal

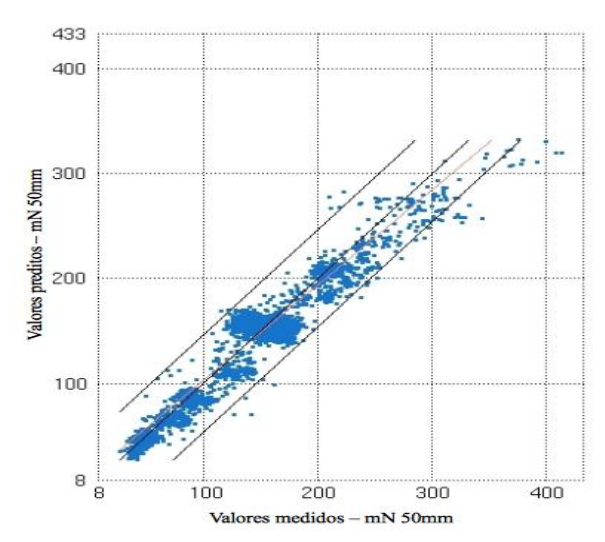


Gráfico 3: Setor 7 - Rigidez Longitudinal

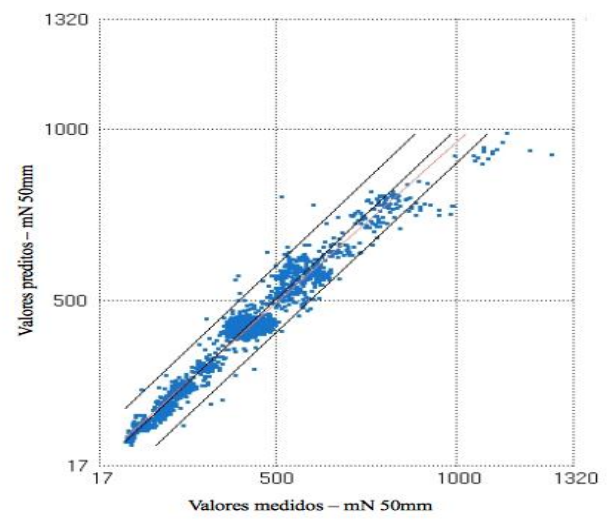

Gráfico 4: Setor 7 - Rigidez Transversal

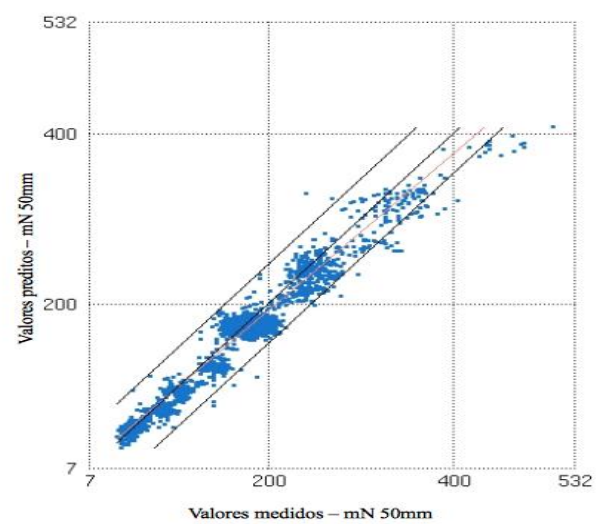

Gráfico 5: Setor 11 - Rigidez Longitudinal

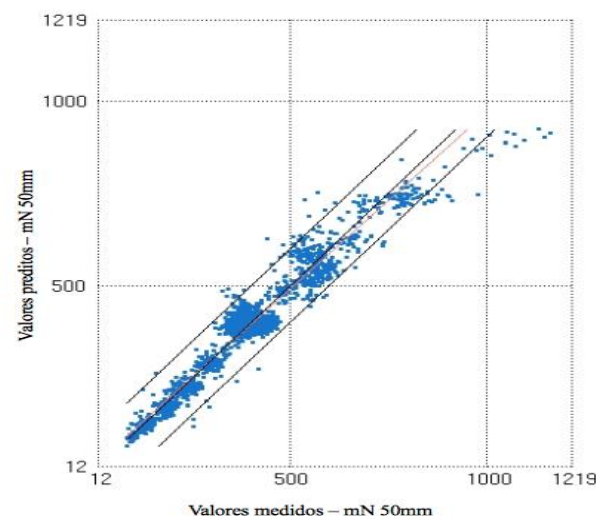

Gráfico 6: Setor 11 - Rigidez Transversal

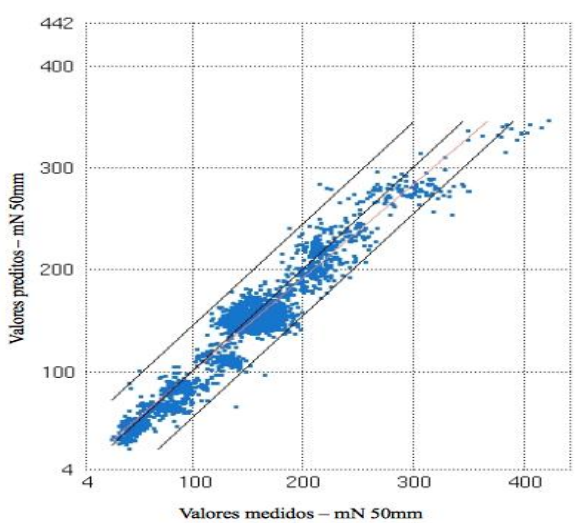

\section{Conclusão}

Todas as redes treinadas apresentaram resultados para o erro relativo com os dados do conjunto de validação menores que 0,35 e $\mathrm{R}^{2}$ maior que 0,88 , o que indica que as redes neurais conseguem predizer o valor da rigidez com boa precisão. Isso permite a construção de um sensor virtual para a rigidez com a utilização de variáveis já existentes no processo (espessura, gramatura e umidade). Um sensor virtual possibilitaria que a produção fosse mantida dentro dos parâmetros estabelecidos reduzindo a geração de produto não conforme e, consequentemente, reduzindo os custos envolvidos. 


\section{Referências}

ASSOCIAÇÃO BRASILEIRA DE CELULOSE E PAPEL. Definição para identificação de papelcartão, cartolinas, papelão e polpa moldada. Disponível em: < http://www. bracelpa.org.br/bra2/?q=node/167 $\geq$.

CASTANHO, C. G.; OLIVEIRA, R. C. Estudos de aproveitamento de rejeito fibroso industrial da polpação kraft de eucalipto na produção de papeis reciclados. CONGRESSO ANUAL DE CELULOSE E PAPEL DA ABTCP, 33, 2000, São Paulo. Anais... São Paulo: ABTCP, 2000.

DRACOPOULOS, D. Evolutionary learning algorithms for neural adaptive control. London: Springer-Verlag London Limited. 1998, cap 4, p. 47-52.

FELLERS, C.; DE RUVO, A.; HTUN, M. et al. Mechanical Properties of Carton Board. In: Carton Board. Profitable use of pulps and processes. Stockholm: STFI, 1983, cap. 6, p. 33-90.

KAJANTO, I. Structural mechanics of paper and board: paper physics. KAARLO, Niskanen. (Ed.). Papermaking science and technology. Jyväskylä: Gummerus Oy, 2000.

KIVIRANTA, A. Paperboards Grades. PAULAPURO, H. Paper and board grades. Papermaking science and technology. Jyväskylä: Gummerus Printing, 2000, cap. 2, p. 54-64.

NISKANEN, K.; PAKARINEN, P. Paper structure: paper physics, KAARLO, Niskanen. (Ed.). Papermaking science and technology. Jyväskylä: Gummerus Oy, 2000.

PAVILION TECHNOLOGIES. Property predictor: user's guide. 1999.

TAPPI. T556pm-95 Provisional method: bending resistance of paper and paperboard (Lorentzen \& Wettre type tester), 1995.
Tabela1- Seleção dos pontos do Sensor

\begin{tabular}{|c|c|c|c|c|}
\hline Setor & Posição & $\begin{array}{c}\text { Folha } \\
6100 \\
\mathrm{~mm}\end{array}$ & $\begin{array}{c}\text { Folha } \\
6200 \mathrm{~mm}\end{array}$ & Adotado \\
\hline \multirow[t]{2}{*}{1} & Início & 319 & 325 & 324 \\
\hline & Final & 329 & 335 & 334 \\
\hline \multirow{2}{*}{2} & Início & 286 & 291 & 288 \\
\hline & Final & 296 & 301 & 298 \\
\hline \multirow{2}{*}{3} & Início & 256 & 260 & 259 \\
\hline & Final & 266 & 270 & 269 \\
\hline \multirow[t]{2}{*}{4} & Início & 222 & 226 & 224 \\
\hline & Final & 232 & 236 & 234 \\
\hline \multirow{2}{*}{5} & Início & 192 & 196 & 194 \\
\hline & Final & 202 & 206 & 204 \\
\hline \multirow[t]{2}{*}{6} & Início & 159 & 162 & 169 \\
\hline & Final & 169 & 172 & 179 \\
\hline \multirow{2}{*}{7} & Início & 129 & 131 & 130 \\
\hline & Final & 139 & 141 & 140 \\
\hline \multirow{2}{*}{8} & Início & 95 & 97 & 96 \\
\hline & Final & 105 & 107 & 106 \\
\hline \multirow{2}{*}{9} & Início & 65 & 66 & 66 \\
\hline & Final & 75 & 76 & 76 \\
\hline \multirow{2}{*}{10} & Início & 31 & 32 & 31 \\
\hline & Final & 41 & 42 & 41 \\
\hline \multirow{2}{*}{11} & Início & 7 & 8 & 8 \\
\hline & Final & 17 & 18 & 18 \\
\hline
\end{tabular}

Figura 1- Divisão da folha para amostragem de rigidez

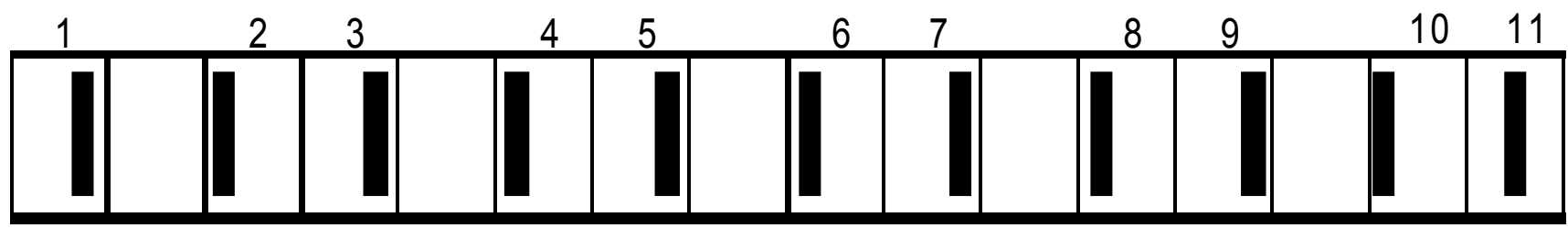


Tabela 2- Resultados dos treinamentos das redes. Erros e $\mathrm{R}^{2}$

\begin{tabular}{|c|c|c|c|c|c|c|c|}
\hline \multirow[t]{2}{*}{ Rede } & \multirow{2}{*}{$\begin{array}{c}\text { Saída } \\
\text { (Rigidez) }\end{array}$} & \multicolumn{2}{|c|}{ Erro Relativo (rel_err) } & \multicolumn{2}{|c|}{$\mathrm{R}^{2}$} & \multicolumn{2}{|c|}{ Desvio Padrão } \\
\hline & & Todos & Validação & Todos & Validação & Todos & Validação \\
\hline \multirow{3}{*}{1} & Longitudinal & 0,232 & 0,170 & 0,946 & 0,971 & 33,353 & 24,448 \\
\hline & Transversal & 0,283 & 0,294 & 0,920 & 0,914 & 15,472 & 16,069 \\
\hline & Todas & 0,239 & 0,190 & 0,943 & 0,964 & & \\
\hline \multirow{3}{*}{2} & Longitudinal & 0,216 & 0,158 & 0,953 & 0,975 & 31,395 & 22,942 \\
\hline & Transversal & 0,251 & 0,302 & 0,937 & 0,909 & 14,276 & 17,185 \\
\hline & Todas & 0,221 & 0,183 & 0,951 & 0,966 & & \\
\hline \multirow{3}{*}{3} & Longitudinal & 0,218 & 0,193 & 0,952 & 0,963 & 32,802 & 28,981 \\
\hline & Transversal & 0,244 & 0,287 & 0,940 & 0,918 & 14,506 & 17,041 \\
\hline & Todas & 0,222 & 0,208 & 0,951 & 0,957 & & \\
\hline \multirow{3}{*}{4} & Longitudinal & 0,211 & 0,167 & 0,955 & 0,972 & 31,929 & 25,151 \\
\hline & Transversal & 0,229 & 0,254 & 0,947 & 0,936 & 14,164 & 15,694 \\
\hline & Todas & 0,214 & 0,182 & 0,954 & 0,967 & & \\
\hline \multirow{3}{*}{5} & Longitudinal & 0,225 & 0,198 & 0,949 & 0,961 & 33,975 & 29,784 \\
\hline & Transversal & 0,239 & 0,235 & 0,943 & 0,945 & 15,123 & 14,867 \\
\hline & Todas & 0,227 & 0,204 & 0,948 & 0,959 & & \\
\hline \multirow{3}{*}{6} & Longitudinal & 0,210 & 0,159 & 0,956 & 0,975 & 31,968 & 24,270 \\
\hline & Transversal & 0,231 & 0,272 & 0,947 & 0,926 & 15,019 & 17,735 \\
\hline & Todas & 0,213 & 0,181 & 0,955 & 0,967 & & \\
\hline \multirow{3}{*}{7} & Longitudinal & 0,213 & 0,161 & 0,955 & 0,974 & 32,498 & 24,527 \\
\hline & Transversal & 0,237 & 0,284 & 0,944 & 0,919 & 15,489 & 18,578 \\
\hline & Todas & 0,217 & 0,186 & 0,953 & 0,966 & & \\
\hline \multirow{3}{*}{8} & Longitudinal & 0,216 & 0,163 & 0,953 & 0,974 & 32,328 & 24,338 \\
\hline & Transversal & 0,232 & 0,251 & 0,946 & 0,937 & 14,819 & 16,027 \\
\hline & Todas & 0,219 & 0,179 & 0,952 & 0,968 & & \\
\hline \multirow{3}{*}{9} & Longitudinal & 0,226 & 0,167 & 0,949 & 0,972 & 32,983 & 24,356 \\
\hline & Transversal & 0,230 & 0,245 & 0,947 & 0,940 & 13,972 & 14,889 \\
\hline & Todas & 0,227 & 0,181 & 0,949 & 0,968 & & \\
\hline \multirow{3}{*}{10} & Longitudinal & 0,237 & 0,188 & 0,944 & 0,965 & 33,642 & 26,718 \\
\hline & Transversal & 0,259 & 0,322 & 0,933 & 0,896 & 14,913 & 18,511 \\
\hline & Todas & 0,240 & 0,212 & 0,942 & 0,955 & & \\
\hline \multirow{3}{*}{11} & Longitudinal & 0,234 & 0,255 & 0,945 & 0,945 & 33,254 & 36,213 \\
\hline & Transversal & 0,268 & 0,342 & 0,928 & 0,883 & 14,890 & 18,994 \\
\hline & Todas & 0,239 & 0,268 & 0,943 & 0,928 & & \\
\hline
\end{tabular}

